\title{
Tongue Tie and Breastfeeding: of the Gap or Another Hype?
}

\author{
Michael AB Naafs* \\ International Health Consultancy, Dutch Internist Endocrinologist, Netherlands
}

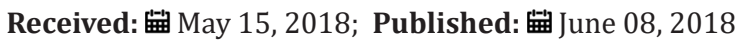

*Corresponding author: Michael AB Naafs, Dutch Internist Endocrinologist, Health Consultant at Naafs,International Health Consultancy, Rhodoslaan 20, 7577KN, Oldenzaal, Netherlands

\begin{abstract}
In this mini-review the pathogenesis,pathohysiology,course and treatment of ankyloglossia or "tongue tie" are discussed in relation to its impact on breastfeeding. Tongue tie surgery has been sky-rocketed last two decades. Pediatric association Campaigns promoting breastfeeding up to the age of 6 months and media attention all contributed.However,the number of operations outpaces the low and weak evidence by far.But young mothers don't wait for further evidence by randomized controlled trials.The number of clinics on internet offering tongue tie surgery to improve breastfeeding rises enormously following the law of demand and supply.
\end{abstract}

\section{Introduction}

Tongue tie (ankyloglossia) is where the strip of skin connecting the baby's tongue to the floor of their mouth is shorter than usual. Some babies who have tongue tie don't seem to be bothered by it. In others it can restrict the tongue's movement,making it harder to breastfeed. Tongue tie is sometimes diagnosed during a baby's routine newborn check but it is not always easy to spot. Tongue tie affects around $4-11 \%$ of newborn babies.It is more common in boys than girls and sometimes runs in families.Babies with tongue tie are not able to open their mouth wide enough to latch on the breast properly.They may have difficulty attaching to the breast or staying attached for a full feed,feed for a long time and be unsettled and seem hungry all the time.They may not gain weight as quickly as they should and can make a "clicking "sound as they feed [13]. Tongue tie can cause problems for a breastfeeding mother as sore or cracked nipples,low milk supply or mastitis. Recently,the subject tongue tie and breastfeeding received much attention in the press and media [4-7]. Tongue tie surgery is sky-rocketing in Australia. During 2006-2016 52.473 Medicare frenotomy items for 0-4 year old children were recorded;the rate increased from 1,22 per 1000 population in 2006 to 6,35 per 1000 in 2016.These data do not capture frenotomies performed by dentists by $\mathrm{CO} 2$ laser frenotomy [8]. Between 1997 and 2012 the U.S. showed an increase in frenotomies of 886\% [9] and Canada (2004-2013) an increase of $89 \%$ [10]. Ths raises the question of whether this surgical management is supported by sufficient evidence $[11,12]$. In this mini-review pathogenesis,pathophysiology,course and treatment of tongue tie will be discussed.

\section{Pathogenesis and pathophysiology}

Ankyloglossia is a congenital anomaly characterized by an abnormally short lingual frenur.Its prevalence in the population is approximately $4 \%$ but varies from 0,02 to $10,7 \%$ in different studies [13-15]. Its definition and severity classification have not yet been clearly established. It mostly appears as a sole anomaly,though it is sometimes accompanied by other congenital anomalies such as cleft palate.As it has a heriditary nature, it occurs more commonly in male children and has been suggested to be related to the X-chromosome [16-18]. A study of the risk factors of ankyloglossia showed that morbidity among infants whose mothers smoked cocaine was three times that among mothers who did not smoke cocaine. Ballard [19] showed that about $21 \%$ of pediatric patients had affected parents [20]. Han showed in a study of 149 Korean children who underwent frenuloplasty an inheritance rate of 20,69 $\%$.X-chromosome mediated inheritance and variation in the gene expression was revealed in the pedigree.The male-female ratio was 2:1. They concluded that ankyloglossia is inherited through X-linked recession and that the mothers of the female patients were carriers [21]. Other environmental factors linked to ankyloglossia and cleft palate are folate deficiency and excess retinoic acid (Vitamin E),a known teratogenic [22]. The link with folate defiiency is however much weaker than in spina bifida .Epigenetic factors may also play 
a role [23]. There is no standard definition of ankyloglossia and multiple classifications exist.When examined the "free tonngue" length in newborns it should be greater than $16 \mathrm{~mm}$.Measurements of less than $11 \mathrm{~mm}$ indicate moderate ankyloglossia and less than $7 \mathrm{~mm}$ indicate severe ankyloglossia.However,this measurement may not be useful in infants. The term posterior ankyloglossia is used when the frenulum is attached at the middle or posterior aspect of the undersurface of the tongue.Taking into consideration the anatomy and function there are many assessment tools for classification.One of them is the Hazelbaker Assessment for Lingual Frenulum Function.This tool uses a scoring system of anatomy and functon [24]. The anatomy part pays attention to the tongue when lifted,elasticity of frenulum,length of lingual frenulum when tongue lifted,attachment of lingual frenulum to tongue and attachment of lingual frenulum to inferior alveolar ridge.The function is viewd as lateralization,lift of tongue,extension of the tongue,spread,cupping of tongue,peristalsis and snapback.It consists of 10 points for frenulum appearance and 14 points for tongue function.

\section{Course of Tongue Tie}

The clinical presentation of symptomatic ankyloglossia varies. The biggest concern as well as research and data collection focus on breastfeeding difficulties that include prolonged feeding,difficulty latching and irritability while feeding. These all lead to poor weight gain.With the American Academy campaign to exclusively breastfeed babies up to 6 months of age,ankyloglossia and its management have gained more attention due to the difficulties when breastfeeding is attempted [25]. There are conerns that ankyloglossia can persist beyond the neonatal period.While some authors describe the possibility of speech related issues due to decreased tongue mobility,others disagree.However,concerned parents frequently ask about future problems with articulation.As the speech develops,some children may exhibit difficulties with the sound of several letters or the combination of letters:l,r,t,d,n.z,th,and sh.It is quite difficult to predict which patients will have articulation problems,or in fact,this association exists [26]. Oher reported problems in patients with ankyloglossia include difficulty eating certain Foods that include licking (ice cream), playing certain wind instruments (flutes,clarinets, tubas and trumpets) and orthodontic problems (open bite and malocclusion) [26]. Dental caries could occur due to food debris not being removed by the tongue's action of sweeping the teeth and spreading of saliva.Spreading of lower incisors with periodontitis and teeth mobility due to long-term tongue thrust are associated problems in untreated ankylglossia [27].

\section{Treatment of Tongue Tie}

Ankyloglossia may be treated with surgical or non-surgical approaches.Surgical modalities include frenotomy,frenulectomy and frenuloplasty.These interventions involve clipping or cutting of the frenulum,generally without sedation.In ancient years this was done by midwives with their finger nails [28]. Laser frenotomy or frenulectomy has also been described and proponents argue that it is more exact and provides better hemostasis than standard frenotomy or frenulectomy.Frenoplasty,is mostly used in older children and adults and refers to rearranging tissue or adding grafts after making incisions and closing the resultant wound in a specific pattern to lengthen the anterior tongue and is most commonly performed under general anaesthesia. There is no sufficient evidence in the literature to favor any one of these 3 main techniques [29]. Frenotomy of the neonate is a simple procedure of less than 2 minutes.Frenectomy and frenuloplasty at or after 6 months of age are performed under general anaesthesia.Revision of the frenum by laser can be performed without a general anaesthetic and revision by electrocautery uses a local anaesthetic. All these methods are equally succesful when used in appropriate circumstances .Non-surgical approaches consist of observation and speech therapy [1-3].

Frenotomy, may be accomplished by incising $2-3 \mathrm{~mm}$ into the lingual frenulum, care being taken not to incise any vascular tissue. There should be minimal blood loss,no more than a drop or two. However,when the procedure is performed by untrained personnel complications can be seriously.These include bleeding,soft tissue injury and infection which can be life- threatening [30]. Laser and electrocautery tecniques offer a safe and effective alternative for scalpel frenectomy operations [31]. If the only goal were to improve breastfeeding,snipping the tie in infancy would be the obvious solution.No anaesthetic is needed,it is relatively cheap,the infants pain is slight,bleeding is negligible and feeding improves immediately.However,when ankyoglossis is associated with foreshortening of the genioglossus muscle,as often occurs,merely snipping the lingual frenulum may not allow free and coordinated movement of the tongue sufficient for the demands of a gradually growing speech and language structure.As a result,further surgery may legitimately needed later. Therefore,the possibility that reevaluation of the situation might become appropriate later,should be emphasized.

\section{Tongue Tie Evidence}

There are few subjects in medicine that have raised so much controversies as the relatively minor and simple surgical treatment of tongue tie in breastfeeding.Consensus on ankyloglossia's role in breastfeeding difficulties is lacking.A minority of surveyed peditricians (10\%) and otolaryngologists (30\%) believes it commonly affects feeding,while $69 \%$ of lactation consultants feel that it frequently causes breastfeeding problems [32]. In a 2016 Cochrane analysis five randomized controlled trials (RCTs; $n=302$ ) met the inclusion criteria [11]. It was concluded that frenotomy reduced breastfeeding mother's nipple pain in the short term. Investigators did not find a consistent positive effect on infant breastfeeding.Researchers reported no serious complications,but the total number of infants was small.The small number of trials along with methodological shortcomings limits the certainty of 
these findings.Further RCTs of high methodological quality are necessary to determine the effects of frenotomy.

A 2016 NICE guidance concluded that there are no major safety concerns about division of ankyloglossia and limited evidence suggests that this procedure can improve breast feeding.This evidence is adequate to support the procedure,provided that normal arrangements are in place for consent, audit and clinical governance [6]. The American Academy of Pediatrics concluded in 2015 that a small body of evidence suggests that frenotomy may be associated with improvements in breastfeeding as reported by mothers, and potentially in nipple pain,but with small,short term studies, inconsistently conducted,strength of evidence is generally low to insufficiënt [1]. Australia's National Health and Medical Research Council's (NHMRC) 2012 Infant Feeding Guidelines state there is limited evidence that tongue tie occurs in appropriately $4-10 \%$ of healthy newborn infants (Evidence Grade D).There is limited evidence (Grade D) to suggest that infants with tongue tie more commonly experience breastfeeding difficulties and further trials are requiered $[8,9]$.

\section{Conclusion}

It is evident the increase in the number of tongue tie operations outpaces evidence It is also clear the increased numbers are likely a reflection of breastfeeding recommendations by pediatric associations [25] and an underlying commitment to helping women breastfeeding and media attention.It is true that classic tongue tie was missed for decades. Unfortunately, the evidence is not there to show that frenotomies improve outcomes. Anyway,young mothers made already their choices and are not waiting for the results of further RCTs of tongue tie and the impact on breastfeeding.The amount of clinics on he internet offering tongue tie surgery has raised enormously following the laws of demand and supply.

\section{References}

1. Francis DO, Kishnaswami S, Mc Pheeters M (2015) Treatment of ankyloglossia and breastfeeding outcomes: a systematic review Pediatrics 135(6): e458-e466.

2. Bin-Nun A, Kasirer YM, Mimoumi FB (2017) A Dramatic Increase in Tongue-Tie Related Articles: A 67 Years Review. Breastfeeding Medicine The Official Journal of the Academy of Breastfeeding Medicine 12(7): 410-414.

3. Power R, Murphy J (2015) Tongue-tie and frenotomy in infants with breastfeeding difficulties: achieving a balance. Arch Dis Child 100(5): 489-494.

4. Wilson CL, Heming MR, Fryer LC (2018) Tongue-ties: a response to media concerns. Oral Surgery 11(1): 88-88.

5. Templeton SK, Gillespie J (2016) Doctors attack "needless" baby tongue surgery. Sunday Times.

6. NICE interventional procedures guidance IPG 149 London. National Institute for Health and Care.

7. Gadd S (2018) Danish parents going abroad for tongue -tie surgery on their infants.CPH Post Onlone.

8. Kapoor V, Douglas PS, Hill PS, Laurence J Walsh,Marc Tennant (2018)
Frenotomy for tongue-tie in Australian children, 2006-2016: an increasing problem. Med J Aust 208(2): 88-89.

9. Walsh J, Links A, Boss E, Tunkel D (2017) Ankyloglossia and lingual frenotomy: national trends in inpatient diagnosis and management in the United States, 1997-2012. Otolaryngol Head Neck Surg 156(4): 735740 .

10. Joseph KS, Kinniburg B, Metcalfe A, Razaz N, Sabr Y, et al. (2016) Temporal trend in ankyloglossia and frenotomy in British Colombia,Canada, 20042013:a population- based study. CAMJ Open 4(1): e33-e40.

11. O'Shea JE, Foster JP, O’Donell CP, Breathnach D, Jacobs SE, et al. (2017) Frenotomy for tongue-tie in newborn infants. Cochrane Database Syst Rev (3): CD011065.

12. Douglas PS, Rethinking (2013) posterior tongue-tie. Breastfeed Med 8: 503-506.

13. Messner AH, Lalakea ML, Aby J, Macmahon J, Bair E (2000) Ankyloglossia:incidence and associated feeding difficulties.Arch. Otolaryngol.Head Neck Surg 126(1): 36-39.

14. Segal LM, Stephonson R, Dawes M, Feldman P (2007) Prevalence,diagnosis and treatment of ankyloglossia:methodoloic review. Can Fam Physician 53(6): 1027-1033.

15. Klockars T, Pitkaranta A (2009) Inheritance of ankyloglossia (tongue tie) Clin Genet 75(1): 98-99.

16. Braybrook C, Doudney K, Marcano AC, Arnason A, Bjornsson A, et al. (2001) The T-box transcription factor gene TBX22 is mutated in X-linked cleft palate and ankyloglossia. Nat Genet 29(2): 179-183.

17. Marcano AC, Doudney K, Braybrook C, R Squires, M Patton, et al. (2004) TBX22 mutations are a frequent cause of cleft palate. J Med Genet 41(1): 68-74.

18. Pauws E, Moore GE, Stanier P (2009) A functional haplotype variant in the TBX22 promoter is associated with cleft palate and ankyloglossis. J Med Genet 46(8): 555-561.

19. Harris EF, Friend GW, Tolley EA (1992) Enhanced prevalence of ankyloglossia with maternal cocaine use.Cleft Palate Craniofac J 29(1): 72-76.

20. Ballard JL, Auer CE, Khoury JC (2002) Ankyloglossia: assessment incidence, and effect of frenuloplasty on the breastfeeding dyad. Pediatrics 110(5): e63.

21. Han SH, Kim MC, Choi YS, Jin-Soo Lim, Ki-Taik Han (2012) A Study on the Genetic Inheritance of Ankyloglossia Based on Pedigree Analysis. Arch Plast Surg 39(4): 329-332.

22. Maldonado E, Lopez-Gordillo Y, Partearrovo T, Varela-Moreiras G, Martínez-Álvarez C, et al. (2018) Tongue Abnormalities Are Associated to a Maternal Folic Acid Deficient Diet in Mice. Nutrients 10(1): 26.

23. Wahl SE, Kennedy AE, Wyatt BH, Moore AD, Pridgen DE, et al. (2010) The role of folate metabolism in orofacial development and clefting. Dev Biol 405(1): 108-122.

24. Amir L, Donath S, James J (2006) Reliabilty of the Hazelbaker Assessment Tool for Lingual Frenulim Function. Int Breastfeeding J 1(1): 3.

25. Eidelman AL (2012) Breastfeeding and the Use of Human Milk: An Analysis of the American Academy of Pediatrics 2012 Breastfeeding Policy Statement. Breastfeeding Medicine 7(5): 323-324.

26. Walsh J, Tinkel D (2017) Diagnosis and Treatment of Ankyloglossia in Newborns and Infants: A Review. JAMA Otolaryngol Head Neck Surg 143(10): 1032-1039.

27. Bhattad MS, Baliga M, Kriplani R (2013) Clinical Guidelines and Management of Ankyloglossia with 1-year Follow up: Report of 3 cases. Case Rep Dent 2013: 185803.

28. Horton CE (1969) Tongue tie Cleft Palate J 6: 8-23. 
29. Tuli A, Singh A (2010) Monopolar diathermy used for the correction of ankyloglossia. J Indian Soc Pedod Prev Dent 28: 130-133.

30. Opara PI, Gabriel-Job N, Opara KO (2012) Neonates presenting with severe complications of frenotomy: a cases series. J Med Case Reports 6: 77.
31. Reddy NR, Marudhappan Y, Devi R, Sumit Narang (2014) Clipping the tongue tie. J Indian Soc Periodontol 18(3): 395-398.

32. Messner AH, Lalakea ML (2000) Ankyloglossia: controversies in management. Int.J.Pediatr. Otorhinolaryngol 54(2-3): 123-131.

\section{(c) \\ i) \\ This work is licensed under Creative Commons Attribution 4.0 License}

To Submit Your Article Click Here:

Submit Article

DOI: 10.32474/MADOHC.2018.02.000142

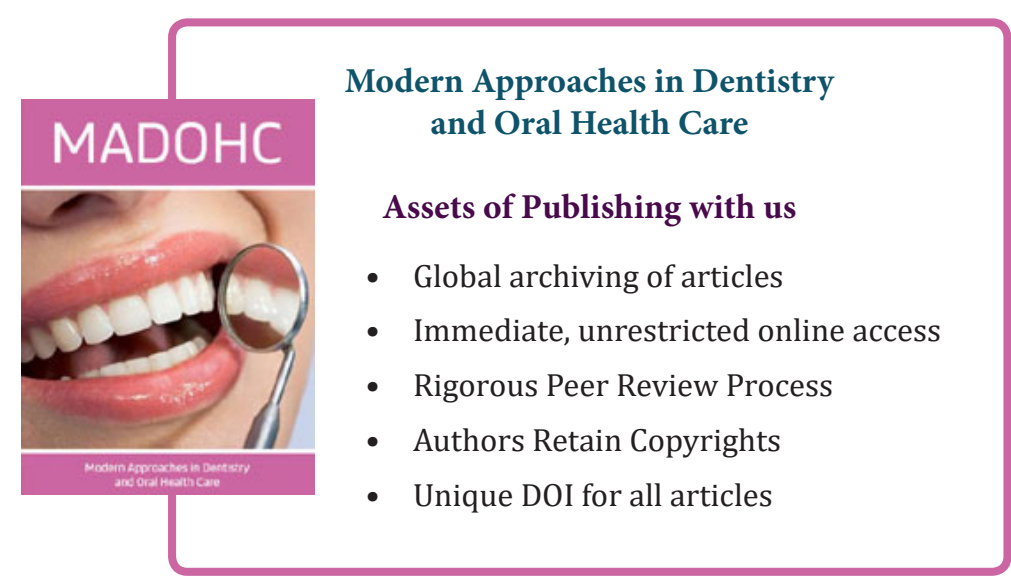

